\title{
Locus architecture affects mRNA expression levels in
}

\section{Drosophila embryos}

Tara Lydiard-Martin, Meghan Bragdon, Kelly B. Eckenrode, Zeba Wunderlich, Angela H.

DePace*

Department of Systems Biology, Harvard Medical School, Boston, MA 02115

* Corresponding author: Angela_DePace@,hms.harvard.edu

\section{Short title:}

Locus architecture affects mRNA levels

Keywords: blastoderm/Drosophila/enhancer/transcription/ 


\begin{abstract}
Structural variation in the genome is common due to insertions, deletions, duplications and rearrangements. However, little is known about the ways structural variants impact gene expression. Developmental genes are controlled by multiple regulatory sequence elements scattered over thousands of bases; developmental loci are therefore a good model to test the functional impact of structural variation on gene expression. Here, we measured the effect of rearranging two developmental enhancers from the even-skipped (eve) locus in Drosophila melanogaster blastoderm embryos. We systematically varied orientation, order, and spacing of the enhancers in transgenic reporter constructs and measured expression quantitatively at single cell resolution in whole embryos to detect changes in both level and position of expression. We found that the position of expression was robust to changes in locus organization, but levels of expression were highly sensitive to the spacing between enhancers and order relative to the promoter. Our data demonstrate that changes in locus architecture can dramatically impact levels of gene expression. To quantitatively predict gene expression from sequence, we must therefore consider how information is integrated both within enhancers and across gene loci.
\end{abstract}

\title{
Introduction
}

How do changes in regulatory DNA sequence impact gene expression? This question is critical for understanding metazoan development, disease and evolution because precise control of gene expression is necessary for the differentiation and function of metazoan cells. Mis-regulation is increasingly implicated in a broad range of disease states (Karczewski et al. 2013; Maurano et al. 2012), and changes in gene expression underlie some morphological differences between animal 
species (Wittkopp et al. 2009; Frankel et al. 2011; Mallarino et al. 2011; Manceau et al. 2011;

Jones et al. 2012). Natural variation in regulatory DNA is common (Mu et al. 2011; Mackay et

al. 2012), but not all changes in regulatory sequence have functional consequences (Romano and

Wray 2003; Hare et al. 2008; Swanson et al. 2011). A central challenge is to learn which and to what extent regulatory sequence variants alter gene expression.

Many classes of cis-regulatory elements that influence metazoan gene expression have been identified, including enhancers, silencers, insulators and targeting sequences (Maston et al. 2006). Cell type specific expression is primarily directed by enhancers that integrate information from multiple DNA-bound transcription factors (TFs) to produce a specific expression pattern (reviewed in Bulger and Groudine 2010). These short ( 1kb) sequences can be located upstream, downstream, or within introns of their target gene. Many genes, particularly key developmental TFs, are regulated by several enhancers that together direct the total gene expression pattern (Levine 2010; de Laat and Duboule 2013). Accordingly, mutation or loss of enhancer sequences can have phenotypic consequences (VanderMeer and Ahituv 2011; Dunipace et al. 2011; Kim et al. 2014).

Natural variation in regulatory sequence spans multiple length scales, from single nucleotide polymorphisms (SNPs) to structural variants such as insertions, deletions, duplications, inversions, and translocations that can range in size from 1-10bp "micro-indels" to $1 \mathrm{Mb}$ (Sudmant et al. 2010; 1000 Genomes Project Consortium et al. 2010; Pang et al. 2010). In humans, structural variation is estimated to account for more than 10 times as much genomic 
variation between individuals as SNPs (Pang et al. 2010). Specific examples of structural variants have been associated with disease (reviewed in Kleinjan and Coutinho 2009) and morphological evolution (e.g., Jones et al. 2012). Structural variants appear to be under strong purifying selective pressure; structural variants in non-coding sequences are selected against more strongly than non-synonymous base substitutions in coding sequences (Zichner et al. 2013).

Despite the prevalence of structural variation, the consequences of large-scale regulatory rearrangements for gene expression have not been systematically studied. Many studies of regulatory sequence variation have focused on the functional impact of SNPs and small indels, either by directed mutagenesis (Thanos and Maniatis 1995; Arnosti et al. 1996; Swanson et al. 2010), or systematic characterization of enhancer variant libraries (Erceg et al. 2014; Melnikov et al. 2012; Kwasnieski et al. 2012; Smith et al. 2013; White et al. 2013). These studies have elucidated how sequence changes within an enhancer impact its regulatory function. Structural variants, meanwhile, may influence the expression of a gene by changing the relative contributions from different enhancers without altering the individual enhancers themselves. Most simply, deleting enhancers can disrupt gene expression (Ludwig et al. 2005; Guenther et al. 2008; Chan et al. 2010; Dunipace et al. 2011; Montavon et al. 2011; McCarroll et al. 2008). Enhancer duplications also impact gene expression, but in unpredictable ways (Klopocki et al. 2008). Rearrangements that move enhancers relative to one another may also alter expression if their bound TFs interact (Small et al. 1993; Kim et al. 2013). Finally, structural variants might disrupt the 3D structure of a locus, which changes during development (Kagey et al. 2010; 
Phillips-Cremins et al. 2013) and is important for the regulation of gene expression (Deng et al.

2012; Dekker et al. 2013).

To investigate how structural variants impact gene expression, we created a set of reporter constructs in which we systematically varied the orientation, order and spacing between two enhancers. TFs are known to interact through short-range and long-range repression mechanisms (Gray and Levine 1996; Courey and Jia 2001; Li and Arnosti 2011), we therefore tested a series of distances between enhancers spanning 0 to $1000 \mathrm{bp}$. We chose to conduct this study in Drosophila melanogaster blastoderm embryos because 1) we could use two well-characterized enhancers from the highly studied even-skipped (eve) locus (Fujioka et al. 1999; Clyde et al. 2003; Struffi et al. 2011); 2) readily integrate our reporters in vivo (Groth et al. 2004); and 3) make quantitative measurements of expression at cellular resolution using fluorescent imaging (Luengo Hendriks et al. 2006; Fowlkes et al. 2008; Wunderlich et al. 2014). This powerful system allowed us to quantitatively probe enhancer activity in the full range of cell types present in developing embryos.

Our results demonstrate that structural variants can have a strong effect on gene expression level. First, contrary to the classic definition of enhancers, we found that levels of expression driven by single enhancers vary with orientation and distance to the promoter; the magnitude and direction of this effect was enhancer-specific. Second, in configurations containing two enhancers, expression pattern position was largely maintained but levels of expression varied by nearly 8fold depending on the orientations, order and spacing of the enhancers relative to one another 
and the promoter. Third, we found that output driven by two enhancers is not equivalent to additive output from the two component enhancers, even when they are separated by a $1000 \mathrm{bp}$ neutral spacer sequence; this indicates enhancers can interact at a much longer range than previously reported. Taken together, our results suggest that structural variants that alter locus architecture are likely to have a substantial impact on gene expression levels. These results emphasize that in order to quantitatively predict gene expression from sequence we must consider how information is integrated at multiple scales - both within enhancers and across gene loci.

\section{Results}

We chose two well-characterized enhancers from the eve locus for our study. eve is expressed in 7 stripes along the anterior posterior axis; these stripes are controlled by 5 separate enhancers (Fujioka et al. 1999). We chose eve 3/7 (which drives expression of stripes 3 and 7) and eve 4/6 (which drives expression of stripes 4 and 6). These two enhancers share regulators (Clyde et al. 2003) and are normally located on opposite sides of the locus (Fig. 1A). We engineered various arrangements of these two enhancers to each other and the promoter using typical reporter constructs that contain the eve basal promoter driving expression of lacZ (Hare et al. 2008). We integrated these reporters into the same genomic location using the phiC31 site-directed integration system (Groth et al. 2004; Fish et al. 2007). When spacer sequence was required, we used portions of the lacZ coding sequence chosen to minimize predicted binding sites for the regulators of these two enhancers (Supplemental Fig. S1 and S2). 


\section{Enhancer distance and orientation relative to the promoter affect target gene expression quantitatively}

We first measured the effect of changing a single enhancer's distance and orientation from the promoter. We cloned the minimal eve 3/7 (511bp; Small et al. 1996) and eve 4/6 (800bp; Fujioka et al. 1999) enhancers at three positions (0bp, 500bp, and 1000bp upstream of the promoter) and in two orientations (either the endogenous orientation, or reversed). We measured the expression from each reporter construct in blastoderm embryos using fluorescent in situ hybridization against the lacZ reporter gene and an endogenously expressed fiduciary marker, fushi-tarazu $(f t z)$. To normalize levels of expression across reporter constructs we co-stained reporter lines with the endogenous gene huckebein $(h k b)$ in the same channel as lacZ (Wunderlich et al. 2014). We imaged entire embryos at cellular resolution and assembled our data into a gene expression atlas, which contains average levels of expression for each gene in each cell for six time points during the hour prior to gastrulation (Luengo Hendriks et al. 2006; Fowlkes et al. 2008). For simplicity, in most figures we show a lateral line trace - the moving average of expression level for a five nuclei wide dorsal-ventral (D/V) strip along the anterior-posterior $(\mathrm{A} / \mathrm{P})$ axis - for the third time point. The full dataset is publicly available at (depace.med.harvard.edu).

We anticipated that these constructs would merely serve as controls for more complex rearrangements of two enhancers relative to one another, but we found that rearrangements of single enhancers have significant effects on the level, but not the position, of expression. Expression level varies by as much as 2-fold across the constructs we tested, both in terms of overall level of expression and the relative expression of the two stripes (Fig. 2 and 
Supplemental Fig. S3). For eve 3/7, expression generally decreases as the enhancer moves away from the promoter, but in the reverse orientation this relationship is not monotonic. In contrast, for eve 4/6, expression increases as the enhancer moves away from the promoter. These results demonstrate that there is a complex relationship between expression level and enhancer position and orientation relative to the promoter.

Despite the changes in expression level, the set of cells expressing the reporter gene was largely consistent in different transgenic lines. After thresholding the gene expression patterns (see Methods), we identified only a handful of cells with statistically significant changes in expression (Fig. 2). For eve 3/7, these cells are associated with variation in expression along the D/V axis. For eve 4/6, the enhancer drives slightly narrower stripes in the reverse orientation at $-1000 \mathrm{bp}$ than when it is in the forward orientation adjacent to the promoter. The qualitative similarity of the expression patterns is consistent with previous studies which found that the stripe enhancers drove expression in the appropriate cells even when moved from their endogenous context to a reporter (Small et al. 1992; 1996; Fujioka et al. 1999). These studies used p-element insertions and were therefore limited to qualitative techniques that could accurately measure expression position, but not level. To fully capture the effects of locus organization on gene expression, cellular resolution quantitative methods are required.

\section{Enhancers do not act independently even when separated by a large spacer sequence}

We next tested how arrangement and spacing of two enhancers relative to one another and the promoter influences expression pattern. We created a set of constructs using eve $3 / 7$, eve $4 / 6$, and 
spacer sequences to systematically test the influence of spacing between enhancers (Fig. 1C). For each spacing we tested several arrangements, labeled A-D, with the spacing indicated by a subscript (Fig. 1D). Our choice of spacing was based on the distance over which short-range repressors can act, because each eve enhancer employs short-range repressors to direct stripe expression (Clyde et al. 2003; Struffi et al. 2011). Short-range repressors bound at one enhancer are capable of disrupting the activity of another enhancer only if placed within 150bp (Fakhouri et al. 2010). We therefore created constructs where the two enhancers are separated by $1000 \mathrm{bp}$, $200 \mathrm{bp}$, and $0 \mathrm{bp}$.

The eve 3/7 and eve 4/6 enhancers are normally on opposite sides of the gene, separated by approximately 9kb, and thought to act additively (Maeda and Karch 2011). We hypothesized that they would still act additively when both are placed upstream of a reporter gene if separated by a sufficiently large neutral spacer sequence. To test this hypothesis, we created a set of four constructs containing the two enhancers upstream of the promoter with a $1000 \mathrm{bp}$ spacer between them, where the orientation and order of the enhancers varies relative to one another and the promoter. Our null expectation was that the output of the two enhancers would simply add together; we calculated this null expectation by adding the expression patterns we measured for each single component enhancer at the properly controlled position and orientation. Comparing the expression patterns driven by our constructs to the null expectation clearly revealed nonadditive behavior that depended on the orientation and arrangement of the enhancers (Fig. 3). The largest discrepancy was for $\mathrm{D}_{1000}$, where expression of stripes 3 and 7 was virtually abolished. In $\mathrm{A}_{1000}, \mathrm{~B}_{1000}$, and $\mathrm{C}_{1000}$ stripe 3 expression increased while stripe 7 did not change, 
indicating that the two stripes do not always change expression in a coordinated way. The eve 4/6 enhancer had lower than expected expression in $\mathrm{A}_{1000}$, and $\mathrm{B}_{1000}$, but increased slightly in $\mathrm{C}_{1000}$. We conclude that enhancer function is sensitive to the presence of other enhancers in the locus and that the underlying mechanism is affected by the position and orientation of the enhancers relative to one another.

To define the range of influence of enhancers on one another's activity, we moved the enhancers closer to one another in the same four configurations. With a 200bp spacer, we observed additional changes in the level of target gene expression compared to constructs with a 1000bp spacer (Fig. 4). Specifically, the reduced expression of eve 4/6 was even more pronounced in $\mathrm{A}_{200}$, and $\mathrm{B}_{200}$, while $\mathrm{D}_{200}$ showed no additional interaction between the two enhancers. In all four configurations, the enhancer closest to the promoter drove lower levels of expression. We conclude that enhancers influence each other's output when they are separated by distances of 200-1000bp, a much longer distance than previously described for interactions between eve 3/7 and eve 2 (Small et al. 1993).

\section{Fused enhancers direct expression patterns only slightly shifted in position}

To quantify the influence of short-range interactions between the two enhancers, we fused them together. We expected interactions between the component enhancers to occur at the junctions, due to local interactions between TFs such as short-range repression and cooperative binding; the four configurations represent all possible junctions between the two enhancers. Previous studies have indicated that short range repression is able to quench activation for up to $150 \mathrm{bp}$ on either 
side of the repressor binding site (Fakhouri et al. 2010; Gray and Levine 1996). Cooperative binding between TFs operates over an even shorter length scale (Crocker et al. 2008; Hanes et al. 1994). Because eve $3 / 7$ and eve $4 / 6$ stripe boundaries are regulated by the same pair of shortrange repressors (Clyde et al. 2003; Struffi et al. 2011; Supplemental Fig. S4), we expected that these TFs would act across the junctions of the fused enhancers, thus changing the position of the stripe boundaries driven by this set of reporters (map of TF binding sites in Supplemental Fig. S1 and S2).

However, when the eve $3 / 7$ and eve $4 / 6$ enhancers were fused together the position of the stripe boundaries changed only slightly in two configurations; expression level was affected in three configurations (Fig. 5). We compared the expression driven by enhancers separated by $200 \mathrm{bp}$ to those directly juxtaposed in order to compare the influence of locus arrangement to the influence of short-range transcription factor interactions. A fusion exhibited no additional changes in expression level or shifts in expression pattern boundaries. The only expression domain that moved was stripe 7; it shifted anteriorly in $\mathrm{C}_{\text {fusion }}(\sim 1$ nucleus width, Supplemental Fig. S5). Expression levels of stripes 4 and 6 were slightly higher in $\mathrm{B}_{\text {fusion }}$ and $\mathrm{C}_{\text {fusion, }}$ and expression in the region of stripe 7 was substantially higher in $\mathrm{B}_{\text {fusion }}$ and slightly increased in $\mathrm{D}_{\text {fusion. }}$. We conclude that interactions between these two enhancers, even at short distances, predominantly affect expression level, rather than the boundaries of expression patterns. 


\section{Levels of gene expression depend on order of enhancers relative to the promoter}

Our data suggest that order of the enhancers relative to the promoter has a significant influence on expression level. The most consistent effect of the enhancer arrangement on target gene expression was a reduction in the level of expression driven by the promoter proximal enhancer. We tested the hypothesis that order relative to the promoter influences the level of expression driven by each enhancer by inverting entire fusions.

Inverting the fusions switched the relative levels of expression driven by the two enhancers. We also observed changes in the relative expression of two stripes driven by the same enhancer (Fig.

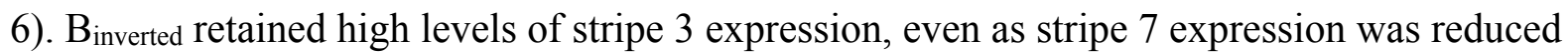
nearly 4-fold. We conclude that order of enhancers relative to the promoter has a strong effect on levels of expression, but that other characteristics, such as orientation, also influence level. In combination with our findings from the single enhancer experiments, these results suggest that distance from the promoter needs to be considered both within enhancers where it manifests as orientation dependence, and across the locus.

\section{Fused enhancers still interact when moved away from the promoter}

In all of our constructs one enhancer was immediately adjacent to the promoter. The promoter may exert an influence on enhancer function, either through chromatin or by the basal transcriptional machinery or associated factors. Many promoters have a well positioned nucleosome upstream of the transcription start site (Mavrich et al. 2008), which might occlude portions of the enhancer. Alternatively, the TFs bound at the promoter proximal enhancer could 
interact directly with the promoter by a different mechanism than when they are farther away. We therefore tested whether moving fused enhancers away from the promoter relieved the repression of the promoter-proximal enhancer.

We found that fusions placed $1000 \mathrm{bp}$ upstream of the promoter still drove the same unequal levels of expression as fusions immediately adjacent to the promoter (Fig. 7). The predominant consequence of moving the fusions away from the promoter was a reduction in the expression in stripes 3 and 7, which is consistent with the observation that the stripe 3/7 enhancer alone had reduced expression when moved away from the promoter. We conclude that depressed expression from the promoter-proximal enhancer does not require a direct juxtaposition of the enhancer and the promoter.

\section{Discussion}

To assess the effect of structural variation on gene regulation, we measured reporter gene expression driven by different arrangements of two eve enhancers in Drosophila melanogaster blastoderm embryos. We systematically varied orientation, order, and spacing of enhancers and measured expression quantitatively at single cell resolution to detect changes in level and position of expression. This approach allowed us to quantify the influence of locus organization while minimizing changes in sequence content. We found that multiple features of locus organization affect expression level significantly; we found only minor changes to the position of the expression pattern. This partially contradicts the classic definition of enhancers as modular units capable of driving the same expression pattern regardless of orientation or distance from 
the promoter and independent of the activity of other enhancers in the locus (reviewed in Maston et al. 2006). First, we found levels of expression driven by single enhancers vary with the enhancer's orientation and distance from the promoter; the direction and strength of this effect is enhancer-specific. Second, we found that in constructs containing two enhancers, levels of expression depend on the order and spacing of the enhancers relative to each other and the promoter. Third, we show that the total expression driven by pairs of enhancers can be nonadditive, even when the enhancers are separated by $1000 \mathrm{bp}$, a much longer range expected for short-range repression mechanisms.

\section{Distance between enhancer and promoter influences expression level}

Here we show that the expression driven by two single eve enhancers is sensitive to the enhancer's position relative to the promoter and this effect is enhancer-specific (Fig. 2B). These results are consistent with experiments using the SV40 enhancer (Wasylyk et al. 1984) and IFNbeta enhanceosome (Nolis et al. 2009), which found that levels of expression driven by these enhancers also depended on distance from the promoter.

One caveat to this experiment is that the sequence adjacent to the 3 ' end of the enhancer changes at each distance due to the different lengths of spacer sequence used. It is thus possible that short-range interactions between transcription factors (TFs) bound near the junction between enhancer and spacer sequence are responsible for the distance dependence that we observe. The degeneracy of eukaryotic transcription factor binding motifs (Wunderlich and Mirny 2009) makes it difficult to completely eliminate all TF binding sites in spacer sequences, and the 
possibility of introducing inappropriate interactions always exists. We examined the predicted TF binding sites in the spacer sequences and found no clear candidates that would explain the observed trends (Supplemental Fig. S1 and S2). The spacer sequences contain few predicted TF binding sites, and there are few activator binding sites near the edges of the enhancers, which we would expect to be most strongly affected by short-range interactions. The introduction of longrange repressor binding sites within the spacer could globally decrease expression (Courey and Jia 2001). However, in this case we would expect the effect of distance to be enhancerindependent since the constructs used the same spacers. Instead, the trends are in opposite directions, which suggests that the distance-dependence is not a function of the spacer sequences.

Enhancer-promoter interactions may differ depending on whether an enhancer is promoterproximal or acting at a distance. Most promoters include a well positioned nucleosome approximately 180bp upstream of the transcription start site (TSS) (Mavrich et al. 2008); when enhancers are in close proximity to the promoter this nucleosome may occlude some binding sites. In yeast, where most regulatory sequences are promoter proximal, nucleosome position has a large effect on which TF binding sites are used (Kim and O'Shea 2008; Raveh-Sadka et al. 2012). In addition, the pre-initiation complex (PIC) containing RNA Pol II, general TFs and cofactors forms a large complex spanning $\sim 100 \mathrm{bp}$ across the TSS, and several components have been found to induce DNA bending (reviewed in Levine et al. 2014). Hence, TFs bound to promoter proximal enhancers can come into direct contact with elements of the PIC (Park and Hong 2012). Conversely, metazoan enhancers commonly act a distance via looping mediated by mediator, cohesin, and TF binding sites in both the enhancer and promoter (Phillips-Cremins et 
al. 2013; Kagey et al. 2010; Su et al. 1991). For example, expression of the $\beta$-globin gene and looping between the locus control region (LCR) and $\beta$-globin promoter are eliminated in GATA1 null cells, but tethering of the two elements with an artificial zinc finger enabled looping and rescued transcription (Deng et al. 2012). The fly sparkling enhancer contains a "remote control element" which is required for the enhancer to drive activity at a distance of 846bp, but not when adjacent to the promoter (Swanson et al. 2010). Taken together, these studies support the idea of direct activation when enhancer and promoter are proximal, and a switch to action at a distance mediated by looping.

How might looping result in different levels of expression than direct interaction between enhancers and promoters? It is possible that once looping is established, the interaction between enhancer, bound TFs, and the PIC is the same as when the enhancer is promoter proximal. Thus, the changes in expression level we observe with enhancer-promoter distance may be due to different frequencies or stabilities of enhancer-promoter interactions. However, it is also possible that acting at a distance allows greater conformational freedom and consequently changes the physical interaction of enhancer bound TFs and the promoter in an enhancer-specific manner.

\section{Orientation of enhancer relative to promoter influences expression level}

The level of expression driven by individual enhancers in our study is sensitive to enhancer orientation. This is particularly evident when the eve $3 / 7$ enhancer is $-500 \mathrm{bp}$ from the promoter (Fig. 2); at this distance the eve 3/7 enhancer drives significantly different levels of expression in each orientation. The relative levels of each stripe driven by a single enhancer (e.g. stripe 3 and 
stripe 7) also vary with both distance and orientation (Supplemental Fig. S3). These data demonstrate that the regulatory sequences that generate each stripe are somewhat separable; the stripes need not change in concert. The location of TF binding sites in the enhancer is asymmetric. The orientation dependence may therefore be due to either different TFs coming into contact with TFs bound to the spacer, or the underlying distance-dependence of the TFs interacting with the promoter. Distance-dependent activity for individual binding sites has been demonstrated in both bacteria (Garcia et al. 2012) and yeast (Sharon et al. 2012). Even in these relatively simple systems with single binding sites the distance dependence function is complex. Enhancers contain many TF binding sites, and the aggregate output if each of those binding sites has distance-dependent activity is hard to predict.

In summary, we suggest that the orientation effect is likely due to a combination of asymmetric distribution of binding sites combined with a dependence on distance from the promoter. At minimum, these experiments demonstrate that the information processing in enhancers is asymmetric and highly sensitive to locus context.

\section{Levels of expression depend on the distance and orientation of enhancers relative to each other and the promoter}

We found that the largest impact on level of expression was due to interactions between two enhancers in the same reporter construct. The classic definition of enhancers as autonomous units led us to formulate the null hypothesis that the two enhancers would have additive outputs. Contrary to our expectation, we observed a large non-additive interaction effect on level of 
expression. Our experiments do not address whether the interaction effect is due to direct physical interaction or indirect interaction, for example, through competition for the promoter. However, we can make some observations about the character of the interaction. The largest effects are correlated with the order of the enhancers relative to the promoter. In general the enhancer closest to the promoter directs lower expression than expected, while the more distant enhancer directs normal or elevated expression (see Fig.s 3, 4 and especially 6). The strength of the interaction is dependent on the distance between the enhancers, for 3 of 4 cases. The exception to this rule is configuration $D_{1000}$, in which the repression of eve $3 / 7$ is extremely strong at all distances tested. The magnitude of this effect is much stronger than the effect of short-range interactions between TFs bound at the junctions between fused enhancers (Fig. 5). Finally, we confirmed that the interaction effect is not due solely to one enhancer being directly adjacent to the promoter (Fig. 7).

One possible explanation for the observed interaction effect is the formation of direct physical interactions between the enhancers. Many TFs recruit co-factors and adapters for the explicit purpose of establishing long range interactions with the promoter (Phillips-Cremins et al. 2013; Kagey et al. 2010; Su et al. 1991), and these may target other enhancers as well. The two enhancers we used share the same regulating TFs but produce different positions of expression due to different sensitivities to the repressors hunchback (hb) and knirps (kni) (Clyde et al. 2003; Struffi et al. 2011). The maintenance of the stripe positions implies that the two enhancers retain separate information integration functions. This constraint argues against the direct interaction of the two enhancers through the formation of a single large complex. 
In addition to activating transcription from the promoter, it has recently been shown that enhancers are themselves transcribed (Kim et al. 2010). Enhancer RNAs (eRNA) are generally short-lived, but a variety of putative functional roles have been assigned to them, including recruitment of co-factors and facilitating looping (reviewed in Lam et al. 2014). In yeast, when two promoters drive expression of a single gene, the upstream promoter is used preferentially because transcription through the downstream promoter disrupts its activity (Hirschman et al. 1988; Iyer and Struhl 1995; Martens et al. 2004). It is possible that the interaction between enhancers that we observe is due to a similar effect in which the eRNA produced by one enhancer interferes with the activity of the other.

An alternate, indirect, form of interaction between enhancers is through chromatin spreading, which is primarily associated with silencing through long-range repression (Courey and Jia 2001; Li and Arnosti 2011). Some enhancers recruit chromatin-modifying enzymes, which alter the chromatin composition of the locus and might produce either silencing or enhancement of nearby enhancers (discussed in Bulger and Groudine 2011). However, this mechanism would be expected to depend only on presence or absence of a second enhancer, not on the relative arrangement of the two. Even if the chromatin spreading was directional, we would expect to see an effect that was more strongly dependent on the orientation of the enhancers rather than order relative to the promoter. 
An intriguing possibility is that the order of enhancers relative to the promoter may influence the 3D structure of the locus and thus the efficiency of enhancer-promoter interactions. Numerous studies have found correlations between enhancer-promoter looping and gene expression (Deng et al. 2012; Chopra et al. 2012). In addition, a study of the hox locus found that enhancers in the locus formed a set of looped contacts even in a transcriptionally silent state supporting the idea that transcriptionally silent enhancer elements regulate the 3D structure of the locus (Montavon et al. 2011). In our constructs the promoter proximal enhancer may be looped out by the distal enhancer, reducing its expression. However, this explanation does not account for increased expression of the distal enhancer. Most likely we are seeing the combined effects of multiple processes, including regulated looping.

It is important to note that the two enhancers in our study drive expression in different sets of cells. The existence of an interaction effect therefore indicates that even when enhancers are transcriptionally silent they can influence one another's output. In differentiating cells, enhancers recruit chromatin modifying activity and may interact with basal transcriptional machinery prior to becoming transcriptionally active (Rada-Iglesias et al. 2011; Creyghton et al. 2010). Our data suggest that "poised" enhancers may influence the activity of neighboring regulatory sequences as well.

\section{Implications for interpreting regulatory sequence variants}

Current computational models focus on predicting the activity of single enhancers and do not take locus-level features into account. Single enhancer models are reasonably successful at 
predicting expression patterns, but do not scale up to the whole locus well (Kim et al. 2013;

Samee and Sinha 2014). Using quantitative methods, we have shown that rearrangements of enhancers may affect target gene expression levels, even when binding site content within the enhancer is maintained. In addition, duplications and deletions are likely to have non-additive effects. Our results suggest that including locus-level parameters beyond TF binding will be necessary for accurate predictions.

\section{Implications for regulatory sequence evolution}

Given our results that locus organization can affect expression level, selection for expression level may explain conservation of locus architecture. A recent population genetics study in Drosophila found that structural variants in both coding and non-coding sequences showed evidence of strong purifying selection (Zichner et al. 2013). Studies in both vertebrates and insects have identified regions of "micro-synteny" in which recombination events are much lower than expected (Sun et al. 2006; Engström et al. 2007; Cande et al. 2009). These regions are enriched for developmental genes and highly conserved elements, a proxy for enhancers. Together, these observations point to an important role for locus architecture in the function of developmental genes.

\section{Materials and Methods}

\section{Construction of reporters and transgenic lines}

We used RedFly to identify coordinates of the eve stripe 3/7 and stripe 4/6 enhancers (Gallo et al. 2011). The eve_stripe_3+7 element is 510bp (Release 5 coordinates 2R:5863006-5863516) 
(Small et al. 1996), while the eve_stripe4_6 element is $800 \mathrm{bp}$ (Release 5 coordinates 2R:

5871404-5872203) (Fujioka et al. 1999). Note that the stripe 4/6 enhancer coordinates from

REDfly contain an extra 208bp on the 3' end compared to the construct tested in Fujioka et al.

(1999). Enhancers were PCR amplified from genomic DNA from $w^{118}$ Drosophila melanogaster

flies and sequence verified. Enhancers were inserted into the multiple cloning site of the pBOY

vector (Hare et al. 2008) using isothermal assembly (Gibson et al. 2009), which leaves scar-less

junctions. LacZ spacer sequences were amplified from the $\mathrm{pBOY}$ vector. $\mathrm{pBOY}$ contains an eve

core promoter $20 \mathrm{bp}$ downstream of the multiple cloning site that drives an eve/lacZ fusion

transcript. The vector also contains an attB site for phiC31 site specific integration (Fish et al.

2007) and the mini-white gene for selection of transformants. Each plasmid was injected into

attP2 flies (Markstein et al. 2008) by Genetic Services, Inc and transgenic flies were

homozygosed using the mini-white eye color marker.

\section{Embryo collection and in situ hybridization}

Embryo collection and whole mount in situ hybridization was performed as previously described (Luengo Hendriks et al. 2006). Briefly, 0-4hr embryos (25C) were collected, dechorionated in $50 \%$ bleach, fixed in a 1:4 mixture of $10 \%$ formaldehyde to heptane, and devitellinized in heptane and methanol by shaking. Embryos were post-fixed in formaldehyde and a formaldehyde based hybridization buffer. Hybridizations were performed at 56C with two or three full length cDNA probes: a DIG-labeled probe for fushi tarazu (ftz), a DNP-labeled lacZ probe and optionally a DNP-labeled probe against huckebein $(h k b)$. The probes were detected by successive antibody staining using anti-DIG-HRP (anti-DIG-POD; Roche, Basil, Switzerland) 
and anti-DNP-HRP (Perkin-Elmer TSA-kit, Waltham, MA, USA), and labeled by reactions with coumarin- and Cy3-tyramide (Perkin-Elmer). Embryos were treated with RNase and incubated with Sytox Green (Invitrogen, Carlsbad, CA, USA) to stain nuclei. Finally, embryos were dehydrated in ethanol and mounted in DePex (Electron Microscopy Sciences, Hatfield, PA, USA), using \#1 coverslips to form a bridge to preserve 3D embryo morphology.

\section{Imaging and image processing}

Embryos were imaged and computationally segmented for further analysis (Luengo Hendriks et al. 2006; Fowlkes et al. 2008). A three-dimensional image stack of each embryo was acquired on a Zeiss LSM Z10 with a plan-apochromat 20x0.8 NA objective using 2-photon microscopy. Embryos were binned into six time points of approximately 10 minute windows using the extent of membrane invagination under phase-microscopy as a morphological marker. Time points correspond to $0-3 \%, 4-8 \%, 9-25 \%, 26-50 \%, 51-75 \%$ and $76-100 \%$ membrane invagination along the side of the embryo that has progressed most. Image files were processed into PointCloud representations containing the coordinates and fluorescence levels for each nucleus. Using the $f t z$ fiduciary marker, PointClouds were registered to an average morphological template to create a gene expression atlas, a summary text file containing the normalized expression level for each reporter construct in each nucleus at each time point.

\section{hkb normalization}

Normalization to a $h k b$ co-stain was performed to test the variation in absolute levels of expression across reporters (Wunderlich et al. 2014). Embryos were stained with a mixture of 
lacZ-DNP and $h k b$-DNP probe. Stains were done in two batches: the first batch contained all single enhancer control lines; the second batch contained all two enhancer constructs and two single enhancer control lines to allow comparison between batches. For each embryo, background was calculated as the mode of the fluorescence distribution. After subtracting background, mean $h k b$ fluorescence was calculated as the geometric mean of the anterior and posterior expression domains. We noted that eve stripe 7 overlaps slightly with the posterior expression domain of $h k b$, and so chose to use the geometric mean of anterior and posterior rather than solely the posterior domain as in (Wunderlich et al. 2014) to limit the impact of overlapping expression. The fluorescence in each nucleus was then divided by the mean $h k b$ fluorescence to yield a normalized expression level.

\section{Data analysis and visualization}

Extraction of lateral line traces, and embryo thresholding were performed in MATLAB using the PointCloud Toolbox (http://bdtnp.lbl.gov/Fly-Net/bioimaging.jsp?w=analysis) and custom scripts. Briefly, lateral line traces are a smoothed moving window average over a 1/16th DV strip (about 5 nuclei wide) along the left side of the embryo. Lateral line traces were taken for each individual embryo after atlas registration and the mean and standard error of the mean was calculated for each point along the AP-axis. To measure positional variation in Fig. 2 individual embryos were thresholded into on/off using the mode+standard deviation of expression values in the trunk (0.2-0.9 egg length) as the threshold. For each cell, the distribution of on/off calls was compared using fexact.m which computes Fisher's Exact Test with permutation (10 times) to control for multiple hypothesis testing. 
To find predicted TF binding sites shown in Supplemental Figures S1 and S2, we used Patser (http://stormo.wustl.edu/software.html), with PWMs as listed in Supplemental Table S1. Background GC content was set to 0.406 , a P-value limit of 0.001 was used. We plotted the predicted binding sites using InSite, an interactive tool developed by Miriah Meyer (http:// www.cs.utah.edu/ miriah/insite/).

\section{Acknowledgments}

The authors wish to thank Ben Vincent, Clarissa Scholes, and Max Staller for insightful discussions and careful editing of the manuscript. We also wish to thank Saurabh Sinha, and Md Abul Hassan Samee for sharing modeling predictions; these results shaped our thinking throughout the project.

\section{References}

1000 Genomes Project Consortium, Abecasis GR, Altshuler D, Auton A, Brooks LD, Durbin RM, Gibbs RA, Hurles ME, McVean GA. 2010. A map of human genome variation from population-scale sequencing. Nature 467: 1061-1073.

Arnosti DN, Barolo S, Levine M, Small S. 1996. The eve stripe 2 enhancer employs multiple modes of transcriptional synergy. Development 122: 205-214. 
Bergman CM, Carlson JW, Celniker SE. 2005. Drosophila DNase I footprint database: a systematic genome annotation of transcription factor binding sites in the fruitfly, Drosophila melanogaster. Bioinformatics 21: 1747-1749.

Bulger M, Groudine M. 2010. Enhancers: the abundance and function of regulatory sequences beyond promoters. Dev Biol 339: 250-257.

Bulger M, Groudine M. 2011. Functional and mechanistic diversity of distal transcription enhancers. Cell 144: 327-339.

Cande J, Goltsev Y, Levine MS. 2009. Conservation of enhancer location in divergent insects. Proceedings of the National Academy of Sciences 106: 14414-14419.

Chan YF, Marks ME, Jones FC, Villarreal G, Shapiro MD, Brady SD, Southwick AM, Absher DM, Grimwood J, Schmutz J, et al. 2010. Adaptive evolution of pelvic reduction in sticklebacks by recurrent deletion of a Pitx1 enhancer. Science 327: 302-305.

Chopra VS, Kong N, Levine M. 2012. Transcriptional repression via antilooping in the Drosophila embryo. Proceedings of the National Academy of Sciences 109: 9460-9464.

Clyde DE, Corado MSG, Wu X, Pare A, Papatsenko D, Small S. 2003. A self-organizing system of repressor gradients establishes segmental complexity in Drosophila. Nature 426: 849853.

Courey AJ, Jia S. 2001. Transcriptional repression: the long and the short of it. Genes Dev 15: $2786-2796$. 
Creyghton MP, Cheng AW, Welstead GG, Kooistra T, Carey BW, Steine EJ, Hanna J, Lodato MA, Frampton GM, Sharp PA, et al. 2010. Histone H3K27ac separates active from poised enhancers and predicts developmental state. Proceedings of the National Academy of Sciences 107: 21931-21936.

Crocker J, Tamori Y, Erives A. 2008. Evolution acts on enhancer organization to fine-tune gradient threshold readouts. PLoS Biol 6: e263.

de Laat W, Duboule D. 2013. Topology of mammalian developmental enhancers and their regulatory landscapes. Nature 502: 499-506.

Dekker J, Marti-Renom MA, Mirny LA. 2013. Exploring the three-dimensional organization of genomes: interpreting chromatin interaction data. Nat Rev Genet 14: 390-403.

Deng W, Lee J, Wang H, Miller J, Reik A, Gregory PD, Dean A, Blobel GA. 2012. Controlling long-range genomic interactions at a native locus by targeted tethering of a looping factor. Cell 149: 1233-1244.

Dunipace L, Ozdemir A, Stathopoulos A. 2011. Complex interactions between cis-regulatory modules in native conformation are critical for Drosophila snail expression. Development 138: $4075-4084$.

Engström PG, Ho Sui SJ, Drivenes O, Becker TS, Lenhard B. 2007. Genomic regulatory blocks underlie extensive microsynteny conservation in insects. Genome Res 17: 1898-1908. 
Erceg J, Saunders TE, Girardot C, Devos DP, Hufnagel L, Furlong EEM. 2014. Subtle changes in motif positioning cause tissue-specific effects on robustness of an enhancer's activity. PLoS Genet 10: e1004060.

Fakhouri WD, Ay A, Sayal R, Dresch J, Dayringer E, Arnosti DN. 2010. Deciphering a transcriptional regulatory code: modeling short-range repression in the Drosophila embryo. Mol Syst Biol 6: 341.

Fish MP, Groth AC, Calos MP, Nusse R. 2007. Creating transgenic Drosophila by microinjecting the site-specific phiC31 integrase mRNA and a transgene-containing donor plasmid. Nat Prot 2: 2325-2331.

Fowlkes CC, Hendriks CLL, Keränen SVE, Weber GH, Rübel O, Huang M-Y, Chatoor S, DePace AH, Simirenko L, Henriquez C, et al. 2008. A quantitative spatiotemporal atlas of gene expression in the Drosophila blastoderm. Cell 133: 364-374.

Frankel N, Erezyilmaz DF, McGregor AP, Wang S, Payre F, Stern DL. 2011. Morphological evolution caused by many subtle-effect substitutions in regulatory DNA. Nature 474: 598-603.

Fujioka M, Emi-Sarker Y, Yusibova GL, Goto T, Jaynes JB. 1999. Analysis of an even-skipped rescue transgene reveals both composite and discrete neuronal and early blastoderm enhancers, and multi-stripe positioning by gap gene repressor gradients. Development 126: $2527-2538$. 
Gallo SM, Gerrard DT, Miner D, Simich M, Soye Des B, Bergman CM, Halfon MS. 2011.

REDfly v3.0: toward a comprehensive database of transcriptional regulatory elements in Drosophila. Nucleic Acids Research 39: D118-23.

Garcia HG, Sanchez A, Boedicker JQ, Osborne M, Gelles J, Kondev J, Phillips R. 2012. Operator sequence alters gene expression independently of transcription factor occupancy in bacteria. Cell Rep 2: 150-161.

Gibson DG, Young L, Chuang R-Y, Venter JC, Hutchison CA, Smith HO. 2009. Enzymatic assembly of DNA molecules up to several hundred kilobases. Nat Meth 6: 343-345.

Gray S, Levine M. 1996. Short-range transcriptional repressors mediate both quenching and direct repression within complex loci in Drosophila. Genes Dev 10: 700-710.

Groth AC, Fish M, Nusse R, Calos MP. 2004. Construction of Transgenic Drosophila by Using the Site-Specific Integrase From Phage $\varphi$ C31. Genetics 166: 1775-1782.

Guenther C, Pantalena-Filho L, Kingsley DM. 2008. Shaping skeletal growth by modular regulatory elements in the Bmp5 gene. PLoS Genet 4: e1000308.

Hanes SD, Riddihough G, Ish-Horowicz D, Brent R. 1994. Specific DNA recognition and intersite spacing are critical for action of the bicoid morphogen. Mol Cell Biol 14: 33643375. 
Hare EE, Peterson BK, Iyer VN, Meier R, Eisen MB. 2008. Sepsid even-skipped enhancers are functionally conserved in Drosophila despite lack of sequence conservation. PLoS Genet 4: e1000106.

Hirschman JE, Durbin KJ, Winston F. 1988. Genetic evidence for promoter competition in Saccharomyces cerevisiae. Mol Cell Biol 8: 4608-4615.

Iyer V, Struhl K. 1995. Mechanism of differential utilization of the his3 TR and TC TATA elements. Mol Cell Biol 15: 7059-7066.

Jones FC, Grabherr MG, Chan YF, Russell P, Mauceli E, Johnson J, Swofford R, Pirun M, Zody MC, White S, et al. 2012. The genomic basis of adaptive evolution in threespine sticklebacks. Nature 484: 55-61.

Kagey MH, Newman JJ, Bilodeau S, Zhan Y, Orlando DA, van Berkum NL, Ebmeier CC, Goossens J, Rahl PB, Levine SS, et al. 2010. Mediator and cohesin connect gene expression and chromatin architecture. Nature 467: 430-435.

Karczewski KJ, Dudley JT, Kukurba KR, Chen R, Butte AJ, Montgomery SB, Snyder M. 2013. Systematic functional regulatory assessment of disease-associated variants. Proceedings of the National Academy of Sciences 110: 9607-9612.

Kim A-R, Martinez C, Ionides J, Ramos AF, Ludwig MZ, Ogawa N, Sharp DH, Reinitz J. 2013. Rearrangements of 2.5 kilobases of noncoding DNA from the Drosophila even-skipped locus define predictive rules of genomic cis-regulatory logic. PLoS Genet 9: e1003243. 
Kim HD, O'Shea EK. 2008. A quantitative model of transcription factor-activated gene expression. Nature structural \& molecular biology 15: 1192-1198.

Kim MJ, Oksenberg N, Hoffmann TJ, Vaisse C, Ahituv N. 2014. Functional characterization of SIM1-associated enhancers. Hum Mol Genet 23: 1700-1708.

Kim T-K, Hemberg M, Gray JM, Costa AM, Bear DM, Wu J, Harmin DA, Laptewicz M, Barbara-Haley K, Kuersten S, et al. 2010. Widespread transcription at neuronal activityregulated enhancers. Nature 465: 182-187.

Kleinjan D-J, Coutinho P. 2009. Cis-ruption mechanisms: disruption of cis-regulatory control as a cause of human genetic disease. Brief Funct Genomic Proteomic 8: 317-332.

Klopocki E, Ott C-E, Benatar N, Ullmann R, Mundlos S, Lehmann K. 2008. A microduplication of the long range SHH limb regulator (ZRS) is associated with triphalangeal thumbpolysyndactyly syndrome. J Med Genet 45: 370-375.

Kwasnieski JC, Mogno I, Myers CA, Corbo JC, Cohen BA. 2012. Complex effects of nucleotide variants in a mammalian cis-regulatory element. Proceedings of the National Academy of Sciences 109: 19498-19503.

Lam MTY, Li W, Rosenfeld MG, Glass CK. 2014. Enhancer RNAs and regulated transcriptional programs. Trends Biochem Sci 39: 170-182.

Levine M. 2010. Transcriptional enhancers in animal development and evolution. Curr Biol 20: R754-63. 
Levine M, Cattoglio C, Tjian R. 2014. Looping back to leap forward: transcription enters a new era. Cell 157: 13-25.

Li LM, Arnosti DN. 2011. Long- and short-range transcriptional repressors induce distinct chromatin States on repressed genes. Curr Biol 21: 406-412.

Ludwig MZ, Palsson A, Alekseeva E, Bergman CM, Nathan J, Kreitman M. 2005. Functional evolution of a cis-regulatory module. PLoS Biol 3: e93.

Luengo Hendriks CL, Keränen SVE, Fowlkes CC, Simirenko L, Weber GH, DePace AH, Henriquez C, Kaszuba DW, Hamann B, Eisen MB, et al. 2006. Three-dimensional morphology and gene expression in the Drosophila blastoderm at cellular resolution I: data acquisition pipeline. Genome Biol 7: R123.

Mackay TFC, Richards S, Stone EA, Barbadilla A, Ayroles JF, Zhu D, Casillas S, Han Y, Magwire MM, Cridland JM, et al. 2012. The Drosophila melanogaster Genetic Reference Panel. Nature 482: 173-178.

Maeda RK, Karch F. 2011. Gene expression in time and space: additive vs hierarchical organization of cis-regulatory regions. Curr Opin Genet Dev 21: 187-193.

Mallarino R, Grant PR, Grant BR, Herrel A, Kuo WP, Abzhanov A. 2011. Two developmental modules establish 3D beak-shape variation in Darwin's finches. Proceedings of the National Academy of Sciences 108: 4057-4062. 
Manceau M, Domingues VS, Mallarino R, Hoekstra HE. 2011. The developmental role of Agouti in color pattern evolution. Science 331: 1062-1065.

Markstein M, Pitsouli C, Villalta C, Celniker SE, Perrimon N. 2008. Exploiting position effects and the gypsy retrovirus insulator to engineer precisely expressed transgenes. Nat Genet 40: $476-483$.

Martens JA, Laprade L, Winston F. 2004. Intergenic transcription is required to repress the Saccharomyces cerevisiae SER3 gene. Nature 429: 571-574.

Maston GA, Evans SK, Green MR. 2006. Transcriptional regulatory elements in the human genome. Annu Rev Genomics Hum Genet 7: 29-59.

Maurano MT, Humbert R, Rynes E, Thurman RE, Haugen E, Wang H, Reynolds AP, Sandstrom R, Qu H, Brody J, et al. 2012. Systematic localization of common disease-associated variation in regulatory DNA. Science 337: 1190-1195.

Mavrich TN, Jiang C, Ioshikhes IP, Li X, Venters BJ, Zanton SJ, Tomsho LP, Qi J, Glaser RL, Schuster SC, et al. 2008. Nucleosome organization in the Drosophila genome. Nature 453: $358-362$.

McCarroll SA, Huett A, Kuballa P, Chilewski SD, Landry A, Goyette P, Zody MC, Hall JL, Brant SR, Cho JH, et al. 2008. Deletion polymorphism upstream of IRGM associated with altered IRGM expression and Crohn's disease. Nat Genet 40: 1107-1112. 
Melnikov A, Murugan A, Zhang X, Tesileanu T, Wang L, Rogov P, Feizi S, Gnirke A, Callan CG, Kinney JB, et al. 2012. Systematic dissection and optimization of inducible enhancers in human cells using a massively parallel reporter assay. Nature Biotechnology 30: 271-277.

Montavon T, Soshnikova N, Mascrez B, Joye E, Thevenet L, Splinter E, de Laat W, Spitz F, Duboule D. 2011. A regulatory archipelago controls Hox genes transcription in digits. Cell 147: 1132-1145.

Mu XJ, Lu ZJ, Kong Y, Lam HYK, Gerstein MB. 2011. Analysis of genomic variation in noncoding elements using population-scale sequencing data from the 1000 Genomes Project. Nucleic Acids Research 39: 7058-7076.

Nolis IK, McKay DJ, Mantouvalou E, Lomvardas S, Merika M, Thanos D. 2009. Transcription factors mediate long-range enhancer-promoter interactions. Proceedings of the National Academy of Sciences 106: 20222-20227.

Pang AW, MacDonald JR, Pinto D, Wei J, Rafiq MA, Conrad DF, Park H, Hurles ME, Lee C, Venter JC, et al. 2010. Towards a comprehensive structural variation map of an individual human genome. Genome Biol 11: R52.

Park KW, Hong J-W. 2012. Mesodermal repression of single-minded in Drosophila embryo is mediated by a cluster of Snail-binding sites proximal to the early promoter. BMB Rep $\mathbf{4 5}$ : $577-582$. 
Phillips-Cremins JE, Sauria MEG, Sanyal A, Gerasimova TI, Lajoie BR, Bell JSK, Ong C-T, Hookway TA, Guo C, Sun Y, et al. 2013. Architectural protein subclasses shape 3D organization of genomes during lineage commitment. Cell 153: 1281-1295.

Rada-Iglesias A, Bajpai R, Swigut T, Brugmann SA, Flynn RA, Wysocka J. 2011. A unique chromatin signature uncovers early developmental enhancers in humans. Nature 470: $279-283$.

Raveh-Sadka T, Levo M, Shabi U, Shany B, Keren L, Lotan-Pompan M, Zeevi D, Sharon E, Weinberger A, Segal E. 2012. Manipulating nucleosome disfavoring sequences allows fine-tune regulation of gene expression in yeast. Nat Genet 44: 743-750.

Romano LA, Wray GA. 2003. Conservation of Endo16 expression in sea urchins despite evolutionary divergence in both cis and trans-acting components of transcriptional regulation. Development 130: 4187-4199.

Samee MAH, Sinha S. 2014. Quantitative modeling of a gene's expression from its intergenic sequence. PLoS Comput Biol 10: e1003467.

Sharon E, Kalma Y, Sharp A, Raveh-Sadka T, Levo M, Zeevi D, Keren L, Yakhini Z, Weinberger A, Segal E. 2012. Inferring gene regulatory logic from high-throughput measurements of thousands of systematically designed promoters. Nature Biotechnology 30: 521-530.

Small S, Arnosti DN, Levine M. 1993. Spacing ensures autonomous expression of different stripe enhancers in the even-skipped promoter. Development 119: 762-772. 
Small S, Blair A, Levine M. 1992. Regulation of even-skipped stripe 2 in the Drosophila embryo. EMBO J 11: 4047-4057.

Small S, Blair A, Levine M. 1996. Regulation of two pair-rule stripes by a single enhancer in the Drosophila embryo. Dev Biol 175: 314-324.

Smith RP, Taher L, Patwardhan RP, Kim MJ, Inoue F, Shendure J, Ovcharenko I, Ahituv N. 2013. Massively parallel decoding of mammalian regulatory sequences supports a flexible organizational model. Nat Genet 45: 1021-1028.

Struffi P, Corado M, Kaplan L, Yu D, Rushlow C, Small S. 2011. Combinatorial activation and concentration-dependent repression of the Drosophila even skipped stripe 3+7 enhancer. Development 138: 4291-4299.

Su W, Jackson S, Tjian R, Echols H. 1991. DNA looping between sites for transcriptional activation: self-association of DNA-bound Sp1. Genes Dev 5: 820-826.

Sudmant PH, Kitzman JO, Antonacci F, Alkan C, Malig M, Tsalenko A, Sampas N, Bruhn L, Shendure J, 1000 Genomes Project, et al. 2010. Diversity of human copy number variation and multicopy genes. Science 330: 641-646.

Sun H, Skogerbø G, Chen R. 2006. Conserved distances between vertebrate highly conserved elements. Hum Mol Genet 15: 2911-2922. 
Swanson CI, Evans NC, Barolo S. 2010. Structural rules and complex regulatory circuitry constrain expression of a Notch- and EGFR-regulated eye enhancer. Dev Cell 18: 359370.

Swanson CI, Schwimmer DB, Barolo S. 2011. Rapid evolutionary rewiring of a structurally constrained eye enhancer. Curr Biol 21: 1186-1196.

Thanos D, Maniatis T. 1995. Virus induction of human IFN beta gene expression requires the assembly of an enhanceosome. Cell 83: 1091-1100.

VanderMeer JE, Ahituv N. 2011. cis-regulatory mutations are a genetic cause of human limb malformations. Dev Dyn 240: 920-930.

Wasylyk B, Wasylyk C, Chambon P. 1984. Short and long range activation by the SV40 enhancer. Nucleic Acids Research 12: 5589-5608.

White MA, Myers CA, Corbo JC, Cohen BA. 2013. Massively parallel in vivo enhancer assay reveals that highly local features determine the cis-regulatory function of ChIP-seq peaks. Proceedings of the National Academy of Sciences 110: 11952-11957.

Wittkopp PJ, Stewart EE, Arnold LL, Neidert AH, Haerum BK, Thompson EM, Akhras S, Smith-Winberry G, Shefner L. 2009. Intraspecific polymorphism to interspecific divergence: genetics of pigmentation in Drosophila. Science 326: 540-544.

Wunderlich Z, Bragdon MD, DePace AH. 2014. Comparing mRNA levels using in situ hybridization of a target gene and co-stain. Methods. 
Wunderlich Z, Mirny LA. 2009. Different gene regulation strategies revealed by analysis of

binding motifs. Trends Genet 25: 434-440.

Zichner T, Garfield DA, Rausch T, Stütz AM, Cannavó E, Braun M, Furlong EEM, Korbel JO.

2013. Impact of genomic structural variation in Drosophila melanogaster based on population-scale sequencing. Genome Res 23: 568-579.

\section{Figure Legends}

Figure 1: Synthetic reporters containing two enhancers from the eve locus test functional consequences of enhancer rearrangements A) The eve locus contains five stripe enhancers encoding the seven stripe pattern of expression in blastoderm embryos. B) We stained embryos for a reporter gene (red) using fluorescent in situ hybridization, and collected image stacks through the entire embryo. We computationally segmented embryos and extracted fluorescence values for each cell, then aligned embryos to an average morphological framework to generate an atlas of average expression patterns (see Materials and Methods). During the hour of development under study the cells are in a sheet on the surface of the embryo and can be represented in 2D as an unrolled cylindrical projection. For simplicity, in most figures we show a subset of our data taken from a line trace through the lateral side of the embryo (grey box). C) We tested synthetic arrangements of two enhancers with different length and positioning of spacers. D) We also tested different configurations of the two enhancers that cover all possible junctions between the two. 
Figure 2: Expression levels driven by the eve 3/7 and 4/6 enhancers depend on enhancer position and orientation relative to the promoter. We measured expression driven by the eve 3/7 (A, B) and eve 4/6 (D, E) enhancers at three distances from the promoter and two orientations as indicated in schematics at top of each panel. We also overlay the measurements for both orientations in order to see the influence of orientation $(C, F)$. We use 99 percentile expression in the trunk (0.2-0.9 egg length) to estimate the level of expression driven by each construct. Expression values were normalized by co-staining with endogenous $h k b$ (see Materials and Methods) to enable comparison across transgenic lines. Individual embryos are shown as grey dots; black bars indicate the mean and 95\% confidence interval of the mean. We observe significant differences in expression dependent on distance and orientation. We also thresholded gene expression in the embryos to test whether the position of expression changed. We show an unrolled embryo view for each distance with the percentage of embryos in which a cell expresses the reporter plotted in blue. Cells that were significantly different from the reference line (0bp from promoter in forward orientation) are plotted in red ( $p<0.05$, Fisher's Exact Test with permutation to control for multiple hypothesis testing). Position does not change for most lines. The most extreme position shift is a narrowing of the stripes in reverse orientation eve $4 / 6$ at $1000 \mathrm{bp}$ from promoter $(\mathrm{E})$.

\section{Figure 3: Some configurations of enhancers separated by $1000 \mathrm{bp}$ produce non-additive}

expression. We created a set of four constructs containing the two enhancers in different orientations and orders relative to one another with a 1000bp spacer sequence between them. We compared each construct to a null hypothesis of additive activity, as illustrated in the schematics 
on the left. Normalized expression as a function of fraction of egg length $(\mathrm{x} / \mathrm{L})$ is shown for lateral line traces of test constructs (red), and the null hypothesis (black). Shadows indicate standard error of the mean (SEM). We also measured the fold-change in mean expression of each stripe relative to the single enhancer controls. We plot the $\log _{2}$ (fold-change) so that increases and decreases in expression appear with comparable magnitudes. Error bars indicate $95 \%$ confidence interval of the mean.

Figure 4: Enhancers influence each other's level when in close proximity. We compared expression from configurations containing a 200bp spacer to $1000 \mathrm{bp}$ spacers and the additive null hypothesis. Normalized expression as a function of fraction of egg length $(\mathrm{x} / \mathrm{L})$ is shown for lateral line traces for configurations with a 200bp spacer (yellow), 1000bp spacer (red), and the null hypothesis (black). Shadows indicate SEM. We also plot the $\log _{2}$ (fold-change) in mean expression of each stripe relative to the single enhancer control for the $200 \mathrm{bp}$ spacer constructs. Expression of stripes 4 and 6 are consistently reduced in configurations A, B and C relative to both the 1000bp spacer version and single enhancer controls.

\section{Figure 5: Local transcription factor interactions have a minor effect on expression from}

fused enhancers. We compared fused enhancers (blue) to the same configuration with a 200bp spacer (yellow) to estimate the influence of local interactions between transcription factors bound at the junction on expression. $\mathrm{B}_{\text {fusion }}$ shows an increase in stripe 7 accompanied by an anterior shift in expression. $\mathrm{C}_{\text {fusion }}$ shows the same shift in stripe 7 without increased expression. Shadows indicate SEM. 
Figure 6: Relative proximity to the promoter influences level of expression driven by

enhancers. We compared the expression of two fusions (dark blue) to a complete inversion of the entire fusion construct (light blue). In both cases, we see a reversal of the relative levels driven by each component enhancer. Stripes 3 and 7 show large changes in level of expression, while the effect on stripes 4 and 6 is smaller. Shadows indicate SEM.

Figure 7: Fused enhancers still interact when moved away from the promoter. We tested the role of the local promoter environment on determining the level of expression of the proximal enhancer by introducing a 1000bp spacer between fused enhancers and the promoter. Expression of fusions at a distance from the promoter (green) is similar to expression adjacent to the promoter (blue), with the exception of eve $3 / 7$, which is lower in configurations A, B and D, consistent with eve 3/7 having lower expression as it moves away from the promoter. Shadows indicate SEM. 
bioRxiv preprint doi: $\mathrm{https} / /$ doi.org/10.1101/005173; this version posted May 14,2014 . The copyright holder for this preprint (which was not certified by peer review) is the author/funder, who has granted bioRxiv a license to display the preprint in perpetuity. It is made available under aCC-BY-NC-ND 4.0 International license.

\section{Lydiard-Martin_Fig1}

A $1 \mathrm{~kb}$

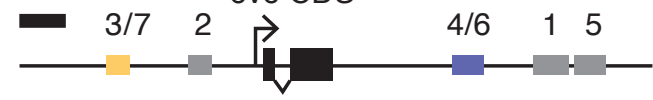

dorsal

ventral

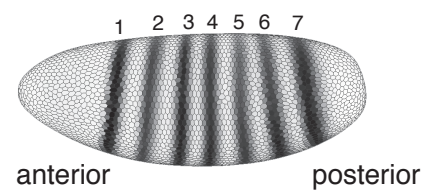

B

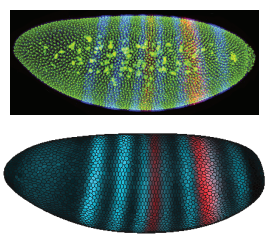

Image stack

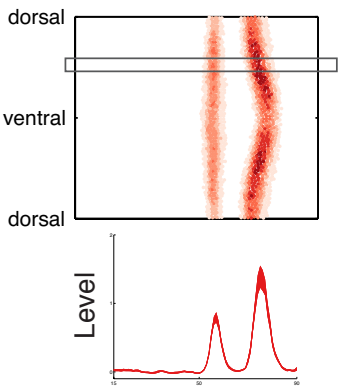

Unrolled

embryo

Line trace

\section{Spacer variations}
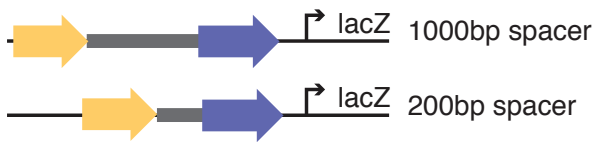

$\longrightarrow$ P lacZ Fusion

C lacZ Inverted Fusion
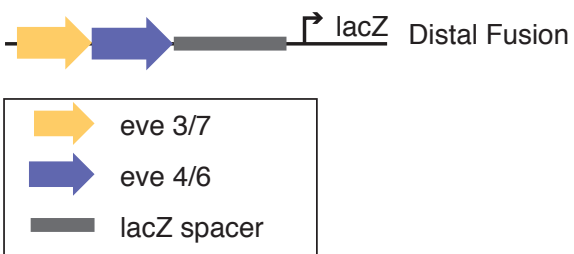

D Configurations
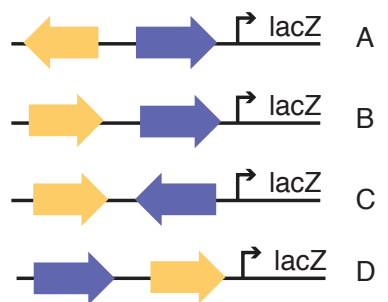


\section{Lydiard-Martin_Fig2}
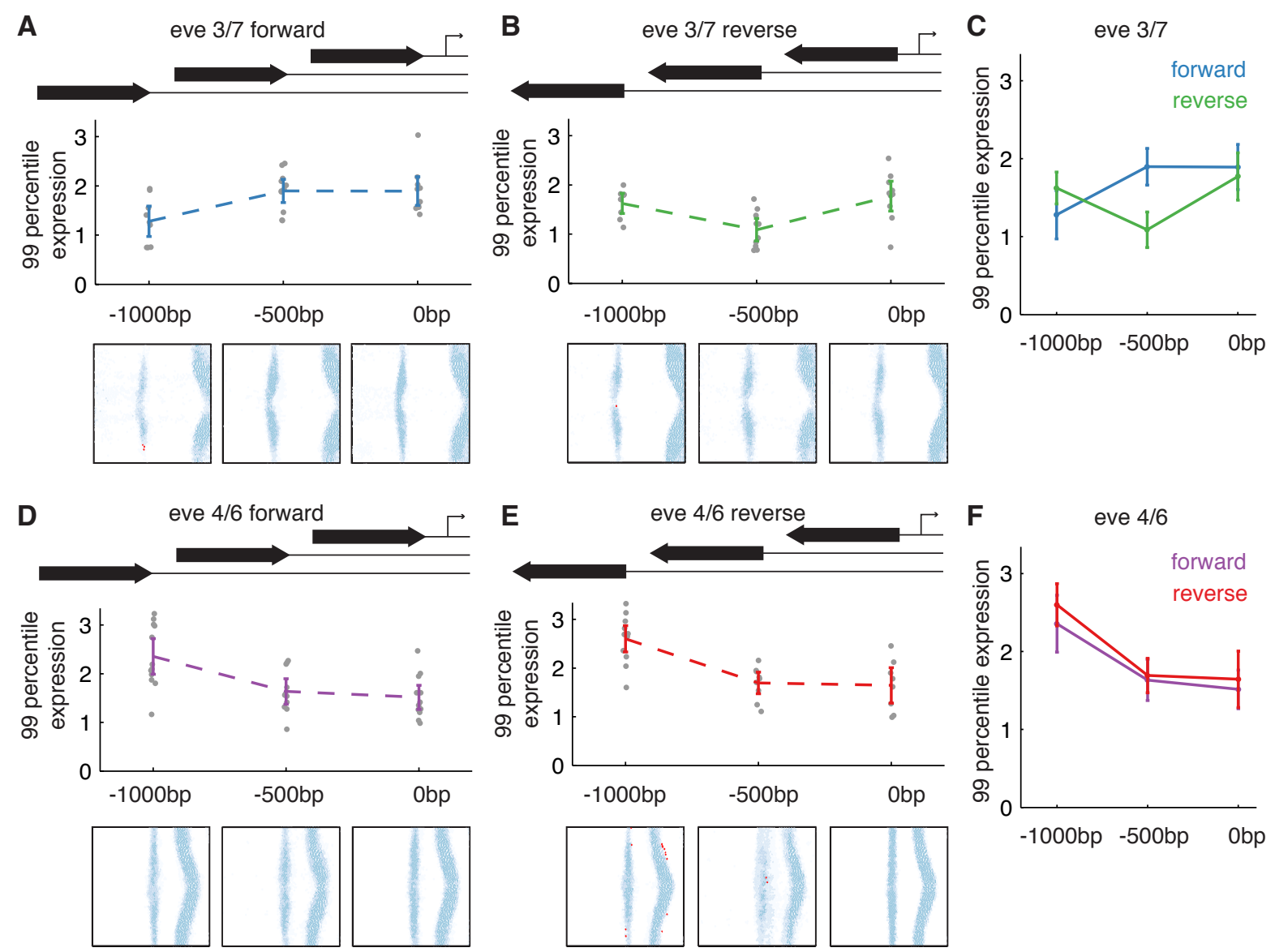
Lydiard-Martin_Fig3
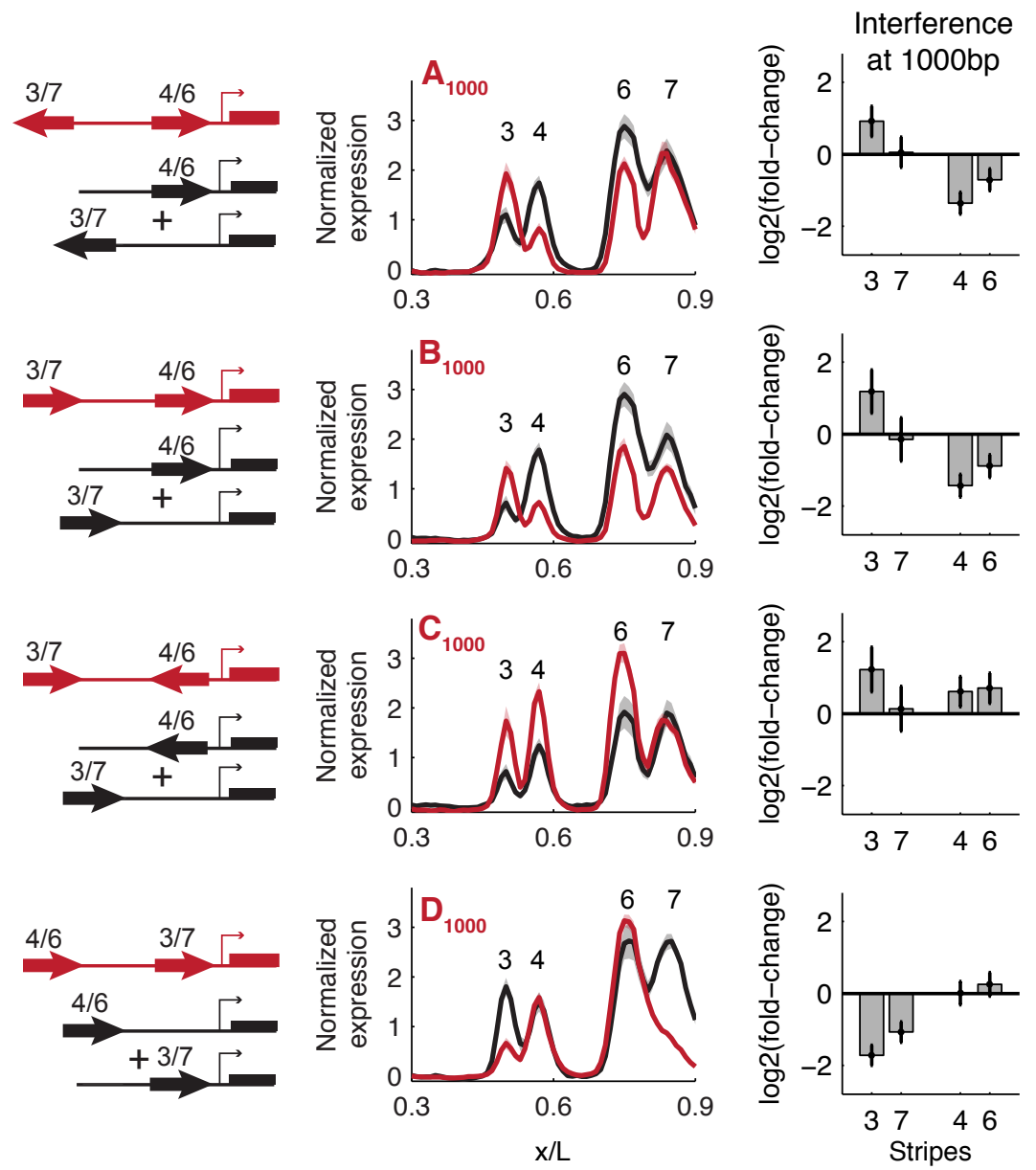


\section{Lydiard-Martin_Fig4}
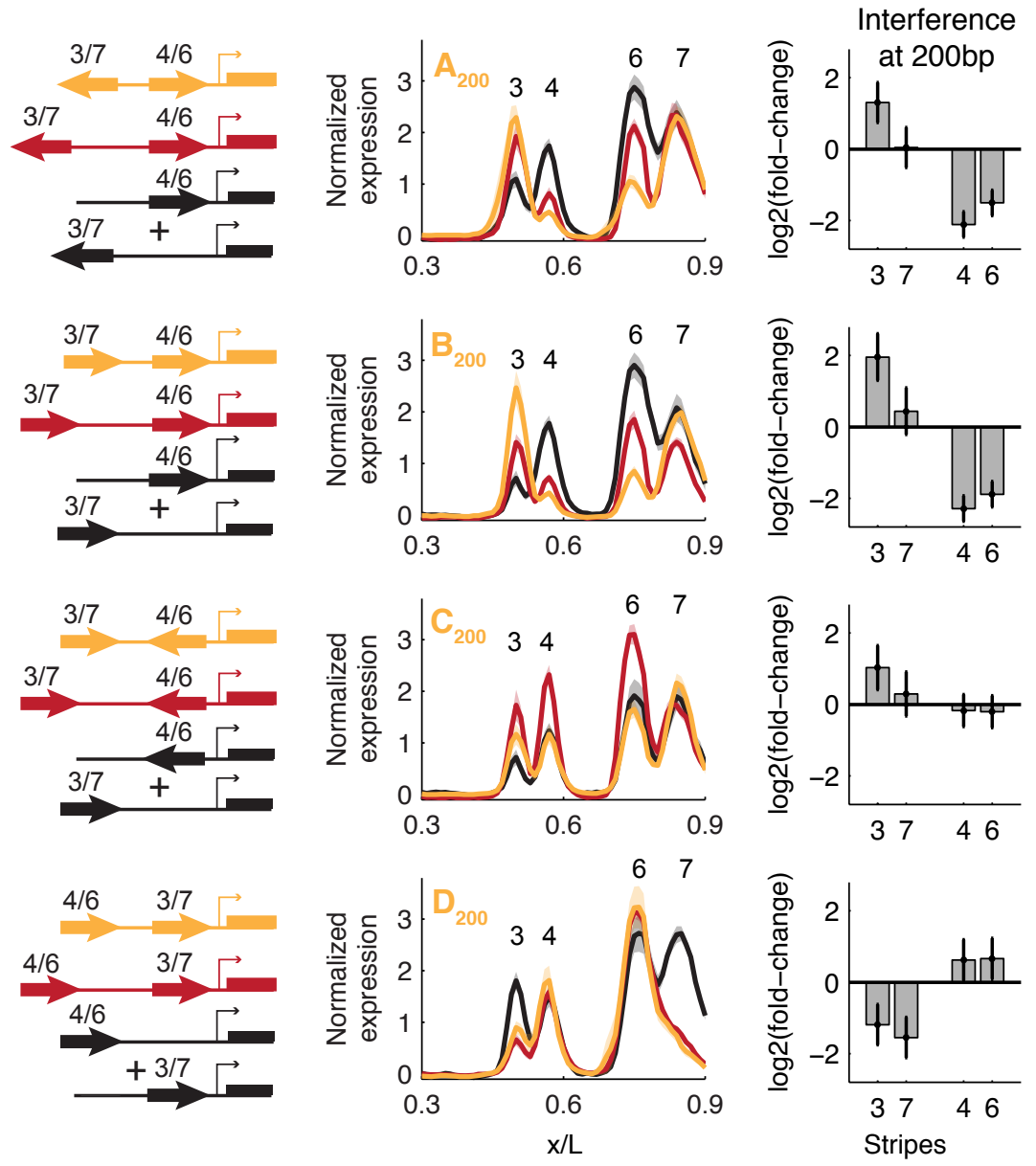
Lydiard-Martin_Fig5
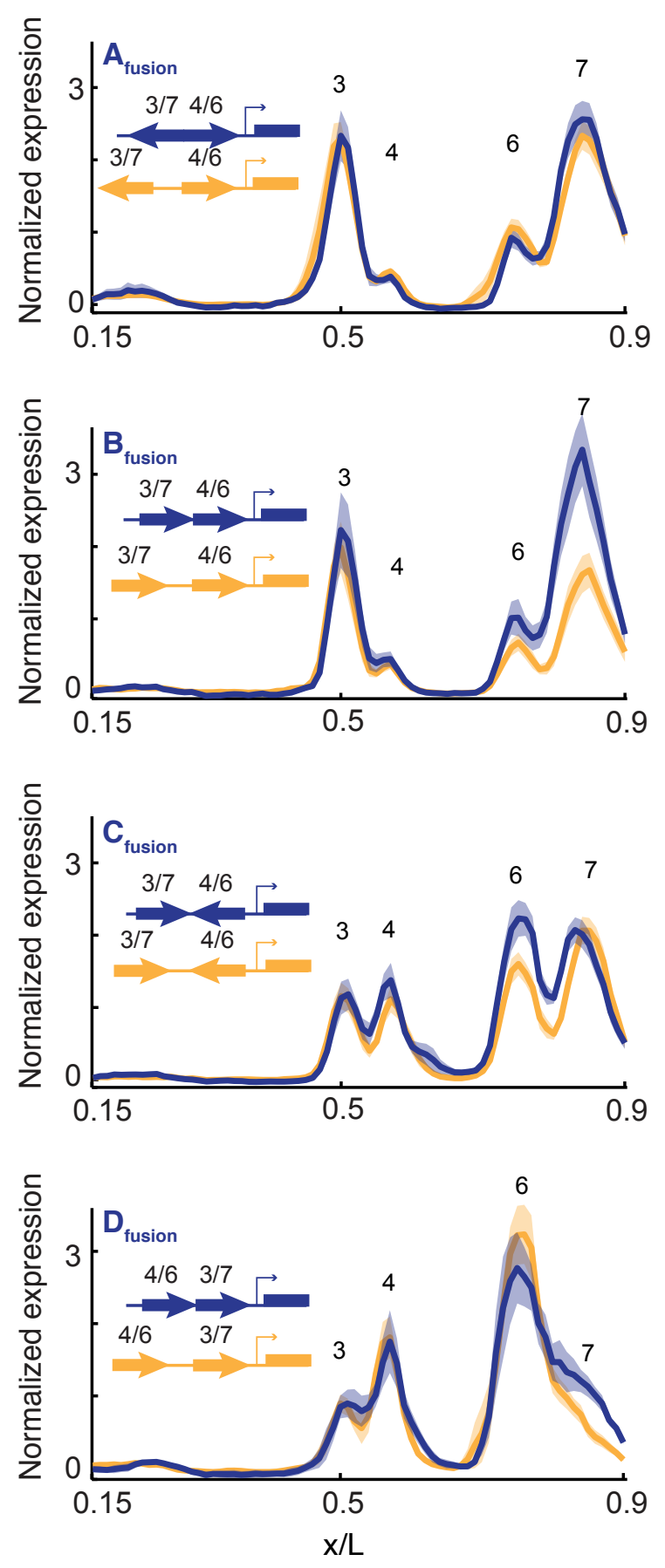
bioRxiv preprint doi: https://doi org/101101/005173: this version posted May 14, 2014. The copyright holder for this preprint (which was not certified by peer review) is the author/funder, who has granted bioRxiv a license to display the preprint in perpetuity. It is made available under aCC-BY-NC-ND 4.0 International license.

Lydiard-Martin_Fig6
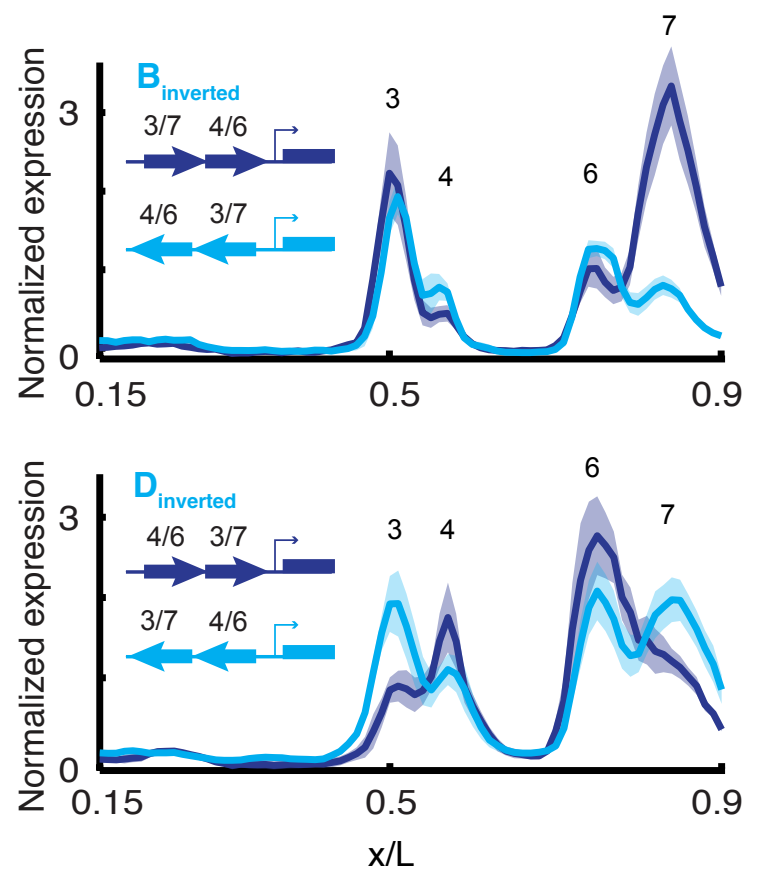


\section{Lydiard-Martin_Fig7}
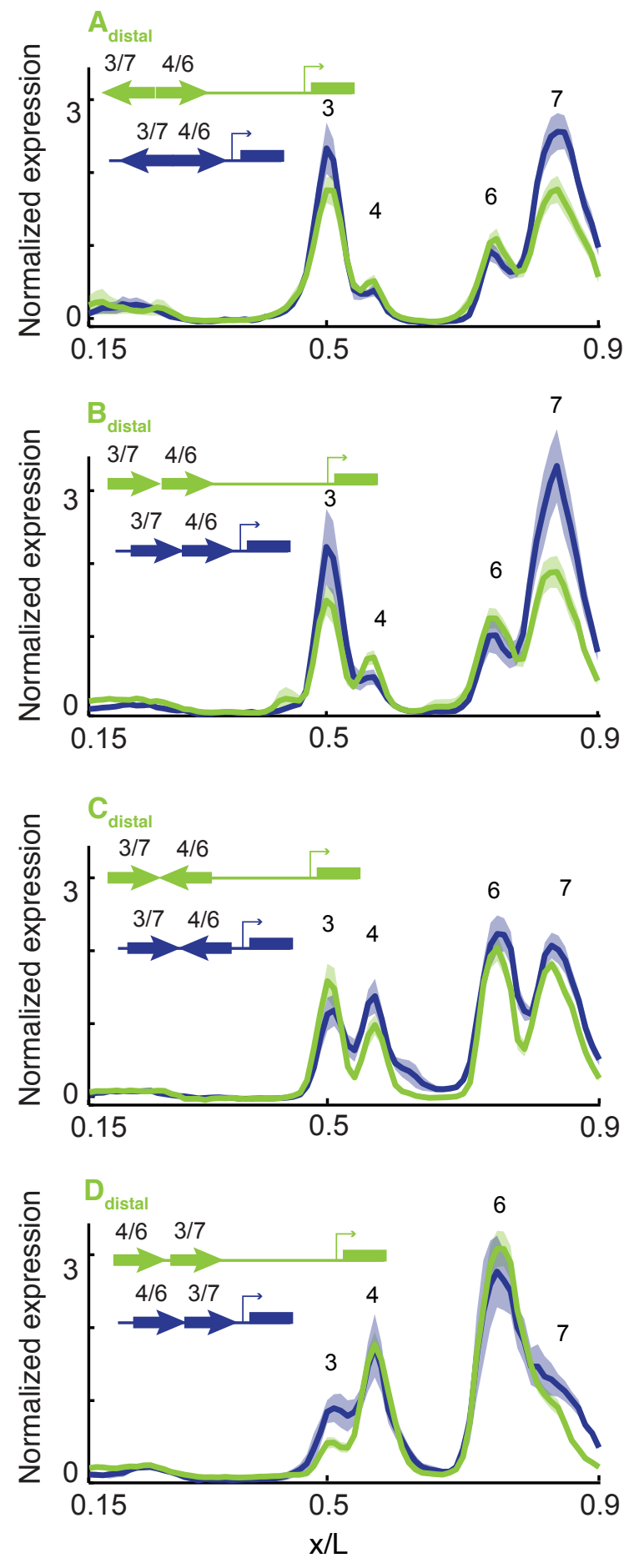